Political Economy Series. Chicago: University of Chicago Press.

Heinz, John P., Edward O. Laumann, Robert L. Nelson, and Robert H. Salisbury. 1993. The Hollow Core: Private Interests in National Policy Making. Cambridge: Harvard University Press.

Hojnacki, Marie, and David C. Kimball. 1998. "Organized Interests and the Decision of Whom to Lobby in Congress." American Political Science Review 92 (4): 775-90.

Kersh, Rogan. 2002. "Washington Lobbyists as Politi- cal Actors." In Interest Group Politics, ed. Allen Cigler and Burdett Loomis. 6th ed. Washington, DC: CQ Press.

Kollman, Ken. 1997. "Inviting Friends to Lobby: Interest Groups, Ideological Bias, and Congressional Committees." American Journal of Political Science 41 (2): 519-44.

Milbrath, Lester W. 1963. The Washington Lobbyists. Chicago: Rand McNally.

Schlozman, Kay Lehman, and John T. Tierney. 1986. Organized Interests and American Democracy. New
York: Harper \& Row.

Wright, John R. 1996. Interest Groups and Congress : Lobbying, Contributions, and Influence. New Topics in Politics Series. Boston: Allyn and Bacon.

Lee Drutman is a Ph.D. candidate in political science at the University of California, Berkeley. His dissertation examines the rise of business lobbying in Washington, $D C$

\title{
Graduate Students Who Attended the Annual Meeting with APSA Assistance
}

$\mathrm{T}$ $\mathrm{n}$ an effort to increase graduate student participation at the Annual Meeting, the association awarded Advanced Graduate Student Travel Grants for the 2010 meeting in Washington, DC. Recognizing challenging economic times across academia, Cambridge University Press offered \$5,000 and Pearson offered \$2,000 to supplement the APSA travel grant program and support U.S. graduate students taking part in the Annual Meeting. The names and institutional affiliates of the awardees follow.

\section{U.S. GRADUATE STUDENTS SUP- PORTED BY CAMBRIDGE UNIVER- SITY PRESS}

Mary Beth Altier, Princeton University Andrew Barwig, University of Denver Shaun Bevan, University of Manchester Constantine Boussalis, Claremont Graduate University

Stephanie Burchard, Rice University Sara Bush, Princeton University Patricia Cavanaugh, Carleton College Ryan Combs, University of Manchester Aaron Hale, University of Florida Matthew Leep, University of Connecticut Ashley Leinweber, University of Florida You-Te Liao, Institute of International Relations

Danielle Lussier, University of California, Berkeley

Christopher McKoy, University of California, Santa Barbara

Amy Nelson, University of California, Berkeley

Sara Newland, University of California, Berkeley

Benjamin Pasquale, New York University

Caryn Peiffer, Claremont Graduate University

Lesley Pruitt, University of Queensland

Brittany Stalsburg, Rutgers University,
New Brunswick

Jonathan Stillo, CUNY-Graduate Center

Laurence Tai, Harvard University

Kevin Walker, Claremont Graduate University

Dana Wittmer, Ohio State University

U.S. GRADUATE STUDENTS SUPPORTED BY PEARSON

Morris Bidjerano, SUNY, Albany

Ashley Conner, Stanford University

Karam Dana, Harvard University

Matthew DiGiuseppe, Binghamton University

Benjamin Graham, University of California, San Diego

William Norris, Massachusetts Institute of Technology

Stephen On, University of California, Los Angeles

Rachelle Suissa, Brooklyn College

\section{U.S. GRADUATE STUDENTS}

Michael Albertus, Stanford University

Gwendoline Alphonso, Cornell University

Ryan Baird, University of Arizona

Amanda Balzer, University of Nebraska, Lincoln

Kiran Banerjee, University of Toronto

Tiffany Barnes, Rice University

Barbra Barnett, Elmhurst College

Kelly Bay, Brown University

Curtis Bell, University of Colorado, Boulder

Philipp Bleek, Georgetown University Janna Bray, University of Michigan Amy Bromsen, Wayne State University Lisa Bryant, University of New Mexico Graham Bullock, University of California, Berkeley

Naomi Choi, University of California, Berkeley

Nick Clark, Indiana University

Meredith Conroy, Occidental College
Adam Cureton, University of North Carolina, Chapel Hill

Allan Dafoe, University of California, Berkeley

Papia Debroy, University of Michigan, Ann Arbor

Christian Donath, University of California, San Diego

Tao Dumas, Louisiana State University

Caitlin Dwyer, University of Minnesota, Twin Cities

Leif-Eric Easley, Harvard University

Andrea Everett, Princeton University

Eleanor Gao, University of Michigan

Michael Georger, Appalachian State University

Kristi Govella, University of California, Berkeley

Erin Graham, Ohio State University

Guy Grossman, Columbia University

Mario Guerrero, University of California, Santa Barbara

Jacqueline Hazelton, Brandeis University

Sara Henary, University of Virginia

David Hendry, University of Illinois at Urbana-Champaign

Alexis Henshaw, University of Arizona

Veronica Herrera, University of California, Berkeley

Catherine Herrold, Duke University

Andrea Jones-Rooy, University of Michigan, Ann Arbor

Eva Kaye-Zwiebel, University of Michigan, Ann Arbor

Jennifer Keister, University of California, San Diego

Athena King, University of South Carolina Brandon Kliewer, University of Georgia

Katrina Kosec, Stanford University

Kyle Kretschman, University of Texas at Austin

Ann Kryzanek, University of Georgia Peter LeQuire, University of Chicago Cyanne Loyle, University of Maryland 
Noam Lupu, Princeton University

Aila Matanock, Stanford University

Lauren McCarthy, University of Wisconsin-Madison

Geoffrey McGovern, Binghamton University

Christopher Meckstroth, University of Chicago

Robbin Mellen, Washington State University

Caitlin Milazzo, University of California, Davis

Michael Miller, Princeton University

Cecilia Mo, Stanford University

Eduardo Moncada, Yale University

Gautam Mukunda, Massachusetts Institute of Technology

William Myers, Michigan State University

Neil Narang, University of California, San Diego

Angela Narasimhan, Syracuse University

Erica Owen, University of Minnesota

Laura Paler, Columbia University

Luke Perez, Villanova University

Timothy Peterson, University of Missouri, Columbia

Jonathan Polk, University of Georgia

Heather Pool, University of Washington

Scott Powell, Ohio State University

David Toohey, University of Hawaii

L. Matthew Vandenbroek, University of Texas at Austin

David Whitney, Louisiana State University

Carla Willis, Ohio State University

INTERNATIONAL GRADUATE STU-

DENTS AT U.S. INSTITUTIONS

Maria Gracia Andia, Northeastern University

Murat Bayar, University of Georgia

Srobana Bhattacharya, Southern Illinois University at Carbondale

CamLy Bui, Syracuse University

Lee Bung-Jae, University of Texas at Austin

Agustin Casas, Northwestern University

Yiftah Elazar, Princeton University

Cengiz Erisen, California Polytechnic State University

Alexander Euler, University of California, San Diego

Paola Fajardo-Heyward, SUNY, Binghamton University

Timothy Fisken, University of California, Berkeley

Anna Getmansky, New York University

Zeynep Goker, CUNY-Graduate Center

Reyko Huang, Columbia University
N. Turkuler Isiksel, Columbia University

Yukari Iwanami, University of Rochester

Eunju Kang, Claremont Graduate University

Ivan Katchanovski, Harvard University

Yujin Kim, University of Wisconsin-Madison

Geun Koh, University of Delaware

Magdalena Krajewska, Brandeis University

Su-Mi Lee, University of Kentucky

Alla Manukyan, Georiga State University

Miwa Nakajo, Texas A\&M University

Kimberly Nolan Garcia, University of New Mexico

Ararat Osipian, Vanderbilt University

Ugur Ozdemir, Washington University in St. Louis

Sunhee Park, Florida State University

Aldo Ponce, University of Houston

Diego Rossello, Northwestern University

Azamat Sakiev, Syracuse University

Ilai Saltzman, Harvard University

Susanne Schorpp, University of South Carolina

Tolga Sinmazdemir, New York University

Julieta Suarez-Cao, Northwestern University

Paul Timmermans, University of Denver

Burcu Ucaray-Mangitli, University of Illinois, Urbana-Champaign

Bradley Verhulst, SUNY, Stony Brook University

Yuhua Wang, University of Michigan

Sami Zeidan, CUNY-Graduate Center

Tong Zhao, Georgia Institute of Technology

\section{INTERNATIONAL SCHOLARS}

Uriel Abulof, Tel-Aviv University

Belgin Akca, Koc University

Gal Ariely, University of Haifa

Umut Aydin, Bogazici University

Magni Beatrice, University of Milan

Jessie Blackbourn, Queen`s University, Belfast

Tobias Boehmelt, ETH Zurich

Alex Braithwaite, University College London

Lucie Cerna, Merton College

Clare Chambers, University of Cambridge

Chun-Chih Chang, National Chengchi University

Titus Chen, University of Southern California

Michael Cohen, University of British Columbia

Alistair Cook, Nanyang Technological University

Jan-Emmanuel De Neve, London School of
Economics

Florin Fesnic, Center for the Study of Democracy, Romania

Erica Frederiksen, University of Toronto

Aina Gallego, Universitat Pompeu Fabra

John Grant, Brock University

Torben Heinze, Freie Universität Berlin

Matthias Hofferberth, Goethe-University Frankfurt

Caroline Hossein, University of Toronto

Timo Idema, University of Oxford

Asaf Kedar, Hebrew University of Jerusalem

Zeynel Kilinc, Sakarya University

Nicholas Kitchen, London School of Economics

Mareike Kleine, London School of Economics

Sergey Kostyaev, Russian Academy of Sciences

Spyridon Kotsovilis, McGill University

Theresa Kuhn, European University Institute

Tomila Lankina, De Montfort University

Joel Lazarus, University of Oxford

James Manicom, University of Toronto

Sofie Marien, KU Leuven

Mihaela Mihai, University of Coimbra

Maya Mitre, Universidade Federal de Minas Gerais

Aurelian Muntean, National School of Political Studies, SNSPA

Francesca Pasquali, Università degli Studi di Milano

Stratos Patrikios, University of Strathclyde

Enrique Peruzzotti, Universidad Torcuato Di Tella

Thamy Pogrebinschi, Instituto Universitário de Pesquisas do Rio de Janeiro (IUPERJ)

Damian Raess, Université de Genève

Ziv Rubinovitz, University of Haifa

Thomas Sattler, University College Dublin

Lee Seymour, Leiden University

Indira Sinha, College of Commerce

Matteo Triossi, Universidad de Chile

Cristian Vaccari, University of Bologna

Srdjan Vucetic, University of Ottawa

Michael Wahman, Lund University

Annemarie Walter, University of Amsterdam

Dong Wang, Peking University

Stan Hok-Wui Wong, Chinese University of Hong Kong

Eric Yu, National Chengchi University (Taipei)

Galina Zudenkova, University Carlos III of Madrid 